Material and Methods During a two year interval, a number of 6 adolescent males were admitted in the ED with severe precordial pain. All were between

14-17 yo. They performed lab investigations, ECG, Echocardiography, Speckle-tracking, Holter ECG and cardiac biomarkers. Two were explored by angiocoronarography due to acute STEMI aspect on ECG, to exclude myocardial infarction. Angio MRI of the heart performed in all patients.

Results Six males were admitted for severe precordial pain, 8 on a scale of $0-10$, which appeared within the last 12 hours, with a duration of 1-2 hours, accompanied by palpitations. One boy had Duchenne disease and one thrombophilia. The CRP was slightly elevated in all cases, with values between 7 and $56 \mathrm{mg} / \mathrm{dl}$; hsCRP was also elevated. CPK was increased in all cases, varying between $134-1834 \mathrm{ng} / \mathrm{ml}$. The highest value was in Duchene patient. CPK-MB was slightly increased in 4 patients and very increased in two. Troponin $\mathrm{T}$ was positive in two patients with values 54 and $51 \mathrm{pg} / \mathrm{ml}$ and increased values in four, between 669 and $2500 \mathrm{pg} / \mathrm{ml}$. Troponin I had very high values, from 501 to $30.000 \mathrm{pg} / \mathrm{ml}$ in one case of multifocal myocarditis.

NTproBNP was altered in all patients, with values between 72 and $1508 \mathrm{pg} / \mathrm{ml}$.

TGO and TGP were elevated, especially TGO. ECG in all cases had the aspect of acute STEMI. Two cases were explored by angiocoronarography with negative result. Ejection fraction was at the lower limit of normal in 3 cases and reduced between $45-49 \%$ in the other three. Speckle-tracking was significant in all 6 cases. Holter ECG detected PVC in 2 cases and bradycardia in one.

Cardiac MRI revealed inflamed heart muscle at late gadolinium enhancement.

MRI was repeated at 6 mo and 1 year after, with good results. Viral serology was negative for: parvovirus, coxackie, adenovirus, cytomegalovirus.

Treatment included: AINS, Carvedilol, diuretic, vitamins, bed rest until troponin normalization and sport avoidance 6 mo to 1 year.

Conclusions Myocarditis in adolescents almost always mimics myocardial infarction. Cardiac biomarkers help in the diagnosis, evolution and follow-up. Troponin I is more accurate than troponin T. The extremely high values of Troponin I was correlated with severe acute multifocal myocarditis. Cardiac MRI with late gadolinium enhancement is the hallmark of myocarditis.

\section{ARRHYTHMOGENIC CARDIOMYOPATHY IN A 15-YEAR OLD BOY WITH DIZZINESS AND SYNCOPE}

Suzana Bitanga*, Matej Katavić, Maja Hrabak Paar, Nikola Krmek, Hrvoje Kniewald. University Hospital Centre Sestre milosrdnice

\subsection{6/archdischild-2021-europaediatrics.174}

Arrhythmogenic cardiomyopathy (arrhythmogenic right ventricular dysplasia) is a hereditary heart muscle disease characterized pathologically by dystrophy and fibrofatty replacement of the right ventricular myocardium, consequently causing ventricle dysfunction and life-threatening cardiac arrhythmias. The right ventricle is predominately affected, but approximately $70 \%$ of hearts studied during autopsy reveal left ventricle involvement. Positive family history is seen in 30\% cases.
Autosomal dominant or autosomal recessive pattern of inheritance are known, latter as part of cardiocutaneous syndromes. The mean age of diagnosis is 30 years, clinical manifestation is rare before puberty. Following hypertrophic cardiomyopathy, it is considered to be the leading cause of sudden cardiac death in adolescency, especially among athletes.

Palpitations, dizziness and syncope can be symptoms of the disease, though sudden cardiac arrest may be the first clinical presentation. Diagnosis is made by combining medical history, ECG, morphological abnormalites (echocardiography and/or cardiac MRI), endomyocardial biopsy and genetic tests. Standardized diagnostic criteria (major and minor), revised in 2010 were made to increase sensitivity and specificity. Treatment options are combination of life-style modification, antiarrhythmic drugs, catheter ablation and ICD implantation.

Here we are illustrating a 15 -year old boy who presented with dizziness and syncope. Cardiac diagnostic workup was performed, revealing arrhythmogenic cardiomyopathy. Inverted $\mathrm{T}$ waves in V1-V4 leads with epsilon waves and premature ventricular contractions with LBBB were noted in standard ECG.

During the 24-hours holter ECG sustained ventricular tachycardia was registered. Echocardiography and cardiac MRI revealed hypertrabeculated right ventricle with regional dyskinesia and akinesia, dyssynchronous right ventricle contractions and additionally fibrous replacement of the right myocardial free wall. ICD was implanted after inadequate antiarrhythmic drugs response.

Conclusion cardiac diagnostic workup should be performed as a severe and progressive heart muscle disease can be the underlying cause od dizziness and syncope.

\section{VERY RARE CASE OF NOONAN SYNDROME, TYPE 2}

LA Gandaeva*, NV Zhurkova, EN Basargina, AA Pushkov, TD Degtayreva, VP Miroshnichenko, OB Kondakova, KV Savostyanov. National Medical Research Center of Children's Health of the Ministry of Health of the Russian Federation, Moscow, Russian Federation

\subsection{6/archdischild-2021-europaediatrics. 175}

Background Noonan syndrome, type 2 (NS2) is rare autosomal recessive disorder of RASopathies group, caused by mutations in the LZTR1 gene. NS2 characterized by a typical face, short stature, broad, short neck, congenital heart disease, developmental delay. The most common heart disease in children with NS2 is hypertrophic cardiomyopathy.

Patients and Methods Patient is a boy, 15 years old with short stature, developmental delay at 1 first year of life and heart disease. He had distinctive facial features of NS: downslanting palpebral fissures, epicanthic folds, hypertelorism, low-set ears short neck, wing-like folds on the neck, pectus deformity. Hypertrophic cardiomyopathy was identified at 1 month old. Now patient have obstructive, hyperthrophic cardiomyopathy, cardiac arrhythmia: ventricular extrasystoles, 4A Lown, intraventricular block combined with bundle branch block, transient WPW. Syncopal episodes.

Surgical correction of obstructive hypertrophic cardiomyopathy: septal myomectomy was performed on the child due to the high risk of developing sudden death syndrome. Target areas of the exome were investigated by next generation sequencing (NGS). Bioinformatic analysis was carried out using ACMG recomendation. Validation of the identified variants was carried out by the Sanger method. 